\title{
Precision strategy based on cancer-specific molecular subtypes of bile duct cancer from comprehensive analysis using multi-omics dataset
}

\author{
Hye Jung $\mathrm{CHO}^{1}$, Incheon $\mathrm{KANG}^{1}$, Sung Hoon $\mathrm{CHOI}^{1}$, Sunyoung $\mathrm{LEE}^{2}$, Ju-Seog $\mathrm{LEE}^{3}$, Sung Hwan $\mathrm{LEE}^{* 1}$ \\ 'Department of Surgery, CHA Bundang Medical Center, CHA University School of Medicine, Seongnam, Korea \\ ${ }^{2}$ Department of Gastrointestinal Medical Oncology, Division of Cancer Medicine, University of Texas MD Anderson Cancer Center, Houston, TX, USA \\ ${ }^{3}$ Department of Systems Biology, Division of Basic Sciences, University of Texas MD Anderson Cancer Center, Houston, TX, USA
}

Introduction: Bile duct cancer is a heterogeneous tumor entity harboring distinct tumor biology and clinical phenotype according to their unique tumor microenvironment, especially anatomic location in terms of intrahepatic, peri-hilar, and distal extrahepatic portion. Therefore, there is a clear unmet need for the precision strategy based on cancer-specific genomic features rather than previous molecular subtypes from bulk RNA sequencing.

Methods: Comprehensive analyses were performed using the dataset from the cancer dependency map project, including cancer-specific molecular characterization with multi-omics data, genome-wide loss-of-function screening with CRISPR-Cas9 system, and drug sensitivity with compound screening to uncover cancer-specific molecular subtypes showing clinical relevance. The cancer-specific molecular signatures were validated in independent translational cohorts (TCGA-CHOL; $\mathrm{n}=45$, ICGC-BTC; $\mathrm{n}=181, \mathrm{GSE} 132305 ; \mathrm{n}=$ 220).

Results: Integrative profiling of transcriptome from bile duct cancer cell lines ( $\mathrm{n}=39$ ) from the Cancer Cell Line Encyclopedia revealed a total of three cancer-specific molecular subtypes showing distinct tumor biology through all omics layer as well as clinical relevance with prognostic significance. Major molecular features of each subtype were reproducible in the validation cohorts. Subtype-specific molecular biomarkers, including mutational signature and metabolites, were identified. Finally, the target drug with subtype-specific genetic dependency was analyzed to provide precision strategy according to distinct subtypes' molecular characterization.

Conclusions: Comprehensive analysis using a multi-omics dataset revealed precision strategies based on cancer-specific molecular subtypes of bile duct cancer in terms of tumor classification and discriminative therapeutic chances. Prospective translational studies companion with clinical trials based on cancer-specific molecular subtypes is mandatory to establish the precision strategy for managing bile duct. 